University of Wollongong

Research Online

Australian Institute for Innovative Materials -

Papers

Australian Institute for Innovative Materials

January 2015

Cascade dynamics of thermomagnetic avalanches in superconducting films with holes

J I. Vestgarden

University of Oslo, j.i.vestgarden@fys.uio.no

F Colauto

Federal University of Sao Carlos

A M H De Andrade

Universidade Federal Do Rio Grande Do Sul

A A M Oliveira

Federal University of Sao Carlos

Wilson Aires Ortiz

Federal University of Sao Carlos

See next page for additional authors

Follow this and additional works at: https://ro.uow.edu.au/aiimpapers

Research Online is the open access institutional repository for the University of Wollongong. For further information contact the UOW Library: research-pubs@uow.edu.au 


\title{
Cascade dynamics of thermomagnetic avalanches in superconducting films with holes
}

\begin{abstract}
The submicrosecond dynamics of thermomagnetic avalanches in superconducting films with nonconducting holes (antidots) is considered. When such an avalanche reaches a hole, it is quickly filled with magnetic flux, and often its rim becomes unstable and a second avalanche is nucleated. In this work the time- and space-resolved behavior of such cascading avalanche behavior is determined using numerical simulations. Results are presented for films with holes of different shape. It is found that holes with sharp corners are those that most frequently create secondary avalanches, and they tend to nucleate in corners.Magneto-optical imaging of $\mathrm{Nb}$ films patterned with the same set of holes strongly supports the numerical results.
\end{abstract}

\section{Keywords}

cascade, superconducting, avalanches, thermomagnetic, holes, dynamics, films

\section{Publication Details}

Vestgarden, J. I., Colauto, F., De Andrade, A. M H., Oliveira, A. A M., Ortiz, W. A. \& Johansen, T. H. (2015). Cascade dynamics of thermomagnetic avalanches in superconducting films with holes. Physical Review B: Condensed Matter and Materials Physics, 92 144510-1-144510-5.

\section{Authors}

J I. Vestgarden, F Colauto, A M H De Andrade, A A M Oliveira, Wilson Aires Ortiz, and Tom H. Johansen 


\title{
Cascade dynamics of thermomagnetic avalanches in superconducting films with holes
}

\author{
J. I. Vestgården, ${ }^{1,2}$ F. Colauto, ${ }^{3}$ A. M. H. de Andrade, ${ }^{4}$ A. A. M. Oliveira, ${ }^{3,5}$ W. A. Ortiz, ${ }^{3}$ and T. H. Johansen ${ }^{1,6}$ \\ ${ }^{1}$ Department of Physics, University of Oslo, P.O. Box 1048 Blindern, 0316 Oslo, Norway \\ ${ }^{2}$ Norwegian Defence Research Establishment (FFI), Kjeller, Norway \\ ${ }^{3}$ Departamento de Física, Universidade Federal de São Carlos, 13565-905 São Carlos, SP, Brazil \\ ${ }^{4}$ Instituto de Física, Universidade Federal do Rio Grande do Sul, 91501-970 Porto Alegre, RS, Brazil \\ ${ }^{5}$ Instituto Federal de Educação, Ciência e Tecnologia de São Paulo, Campus Matão, SP, Brazil \\ ${ }^{6}$ Institute for Superconducting and Electronic Materials, University of Wollongong, Northfields Avenue, Wollongong, NSW 2522, Australia
}

(Received 16 July 2015; published 20 October 2015)

\begin{abstract}
The submicrosecond dynamics of thermomagnetic avalanches in superconducting films with nonconducting holes (antidots) is considered. When such an avalanche reaches a hole, it is quickly filled with magnetic flux, and often its rim becomes unstable and a second avalanche is nucleated. In this work the time- and space-resolved behavior of such cascading avalanche behavior is determined using numerical simulations. Results are presented for films with holes of different shape. It is found that holes with sharp corners are those that most frequently create secondary avalanches, and they tend to nucleate in corners. Magneto-optical imaging of $\mathrm{Nb}$ films patterned with the same set of holes strongly supports the numerical results.
\end{abstract}

DOI: 10.1103/PhysRevB.92.144510

In the critical state of type-II superconductors [1], the metastable system is susceptible to abrupt redistributions of magnetic flux and electrical currents. For thin samples experiencing a perpendicular magnetic field, even tiny field changes may cause large amounts of flux to jump. Typically, such an avalanche lasts less than a microsecond [2,3], and propagates in a fingering structure, often strongly branched, as observed in films of many materials [4-13] using magnetooptical imaging (MOI). These dramatic events are caused by an instability [14] creating a runaway of flux motion accompanied by local heating. Linear stability analysis of the thermomagnetic model has explained why there exist onset threshold values both in applied magnetic field and temperature [15-17]. Numerical simulations [18-21] have confirmed that the equations representing the thermomagnetic scenario of thin superconductors in perpendicular field indeed give rise to the type of dendritic patterns found experimentally.

Interestingly, MOI observations have also revealed that the avalanches show systematic new behavior when meeting macroscopic nonuniformities in the sample. For example, when a layer of normal metal covers part of the superconducting film, an avalanche tends to change propagation direction at the boundary between bare and coated film [22,23]. A recent study of $\mathrm{NbN}$ films coated with a $\mathrm{Cu}$ layer showed that the deflection of the avalanche branches tend to follow Snell's law with a refraction index of 1.4 , a number also found to be the ratio of avalanche propagation velocities in the bare and coated film [24]. Other experiments have shown that inhomogeneities in the form of patterned holes in the superconducting film also modify the avalanche behavior. For example, in films with regular arrays of small holes (antidots), one finds strong guidance of the avalanche branches [25-28]. How avalanches are affected by large holes has also been investigated. In the work of Ref. [29] the process of flux jumping into the central hole of a circular planar ring of $\mathrm{MgB}_{2}$ was studied, revealing major redistribution of the shielding currents. Recently [30], macroholes in $\mathrm{Nb}$ films were considered as potential traps, or stop holes, serving to limit the spatial extension of thermomagnetic avalanches.
PACS number(s): 74.78.-w, 68.60.Dv, 74.25.Ha

The present work aims to reveal the ultrafast cascade dynamics of avalanches propagating in superconducting films patterned with macroholes. Space- and time-resolved results from quantitative numerical simulations for samples with holes of various shapes are presented, and compared with avalanche behavior observed in a similarly patterned superconducting film.

MOI experiments were conducted on a square $\mathrm{Nb}$ film of sides $2 a=2.5 \mathrm{~mm}$ and thickness $d=200 \mathrm{~nm}$. Using optical lithography the film was patterned with holes shaped as a square, a circle, and two triangles [see Fig. 1 (left)]. Each hole has a dimension $0.2 a$, and is placed a distance $0.1 a$ from the nearest edge. To visualize the flux density a ferrite-garnet film with large Faraday coefficient [31] was placed directly on the sample, and after cooling down in an optical cryostat, polarized light microscopy was performed.

The $\mathrm{Nb}$ film was first zero-field cooled to $5 \mathrm{~K}$, a temperature sufficiently low for avalanches to occur. When a transverse magnetic field is gradually applied, flux begins to penetrate, and when reaching $2.5 \mathrm{mT}$ one observes the magneto-optical image shown in Fig. 1 (right). The image was recorded with slightly uncrossed polarizers in the MOI setup, allowing one to discriminate between flux into or out of the image plane. The central part of the film, which is in the Meissner state, serves as a reference color representing $B_{\mathrm{z}}=0$. The bright rim around the sample perimeter shows the applied field piling up outside the diamagnetic film. The image also shows a shallow flux penetration from the edge, in a pattern typical for critical-state behavior. Note that the holes are clearly visible in the image. All four holes display a dark side towards the sample edge, and a bright side towards the center. This bipolar (flux-antiflux) feature is caused by the additional field created by the shielding currents as they are forced to circumvent the holes [32].

When the applied field reaches $2.8 \mathrm{mT}$ the first avalanche event occurs, resulting in the final flux distribution shown in Fig. 2 (left). The avalanche started from the upper edge, and from there invaded the square hole through the thick finger seen to bridge the two regions. Then, from the two inner corners of the square hole secondary events started, resulting in two 

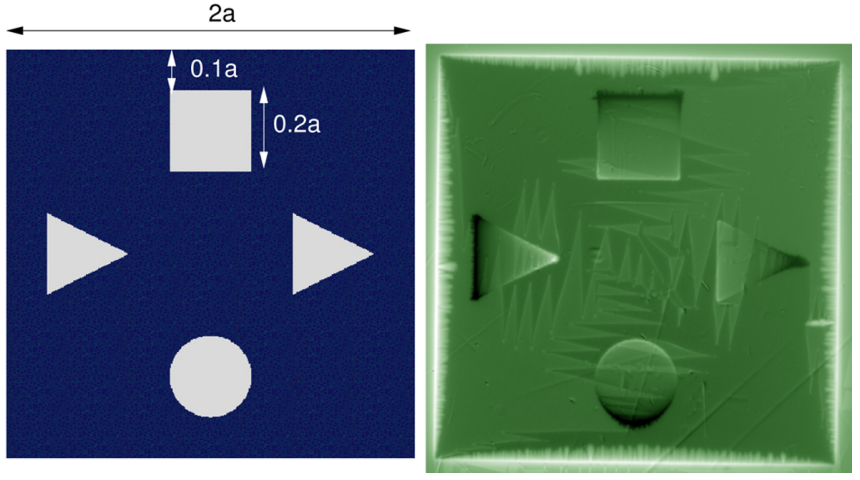

FIG. 1. (Color online) (Left) Sketch of the sample, a square film with four holes. (Right) Magneto-optical image of the perpendicular flux density, $B_{\mathrm{z}}$, in a $\mathrm{Nb}$ film at an applied field below the onset threshold for avalanche activity.

highly branched avalanches. Both of them reached their nearest triangular hole, which by their bright contrast, are seen to have received considerable amounts of flux. Then, as a final step in this cascade of events, the triangular hole on the right side releases its magnetic pressure in an avalanche consisting of three branches.

By increasing the field further, even more avalanches appear, and Fig. 2 (right) shows the flux distribution at the field of $4.35 \mathrm{mT}$. Here, all the holes have received flux, and one sees that some avalanches, e.g., both events starting from the right sample edge do not feed flux into any hole. Continued field ramping adds more and more avalanches, and the complexity in the overall pattern grows [33].

In the following we present results from a numerical simulation of such sequences of events, thus allowing deeper insight into this ultrafast cascading behavior.

The electrodynamics is solved by time integration of the thin-film Maxwell equations

$$
\dot{\mathbf{B}}=-\nabla \times \mathbf{E}, \nabla \times \mathbf{H}=\mathbf{J} \delta(z), \nabla \cdot \mathbf{B}=0,
$$

with $\mu_{0} \mathbf{H}=\mathbf{B}$ and $\nabla \cdot \mathbf{J}=0$, using implicit boundary conditions for edges and holes, as described in Ref. [34]. The material characteristics of the sample are properly described
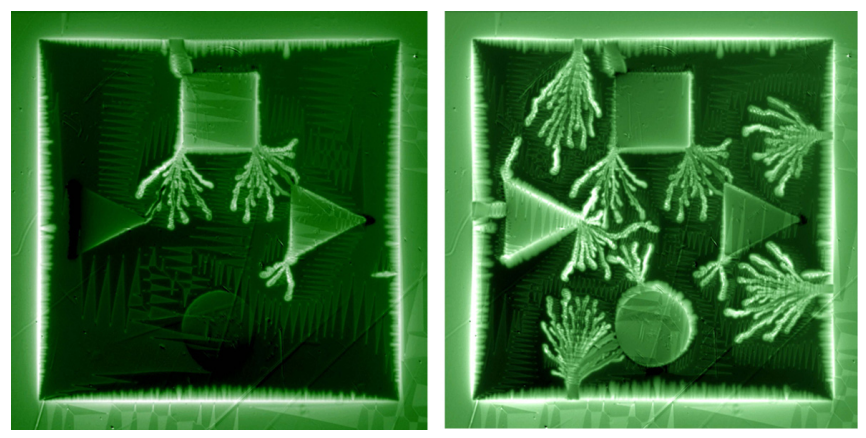

FIG. 2. (Color online) Magneto-optical images of $B_{\mathrm{z}}$ in a $\mathrm{Nb}$ film cooled to $5 \mathrm{~K}$, and then exposed to perpendicular applied fields of $2.8 \mathrm{mT}$ (left) and $4.35 \mathrm{mT}$ (right). The branching patterns in the flux distribution are traces of the flux avalanches. by a conventional power-law $E-J$ relation

$$
\mathbf{E}=\rho_{0} \mathbf{J} / d \begin{cases}1, & |\mathbf{J}|>d j_{\mathrm{c}} \text { or } T>T_{\mathrm{c}} \\ \left(|\mathbf{J}| / d j_{\mathrm{c}}\right)^{n-1}, & \text { otherwise, }\end{cases}
$$

where $T$ is the local temperature, $j_{\mathrm{c}}$ is the critical current density, and $n$ is the creep exponent. The temperature dependencies are $j_{\mathrm{c}}=j_{\mathrm{c} 0}\left(1-T / T_{\mathrm{c}}\right)$ and $n=n_{1} T_{\mathrm{c}} / T$. The local temperature is found by time integration of

$$
c \dot{T}=\kappa \nabla^{2} T-h\left(T-T_{0}\right) / d+J E / d,
$$

where $c$ is the specific heat, $\kappa$ is the heat conductivity, $h$ is the coefficient of heat transfer to the substrate, and $T_{0}$ is the substrate temperature.

The simulation uses the following parameters: $T_{\mathrm{c}}=$ $9.2 \mathrm{~K}, \quad \rho_{0}=5 \times 10^{-9} \Omega \mathrm{m}, \quad j_{\mathrm{c} 0}=1.2 \times 10^{11} \mathrm{~A} / \mathrm{m}^{2}, \quad \kappa=$ $[20 \mathrm{~W} / \mathrm{K} \mathrm{m}]\left(T / T_{\mathrm{c}}\right)^{3}, c=\left[3 \times 10^{4} \mathrm{~J} / \mathrm{K} \mathrm{m}^{3}\right]\left(T / T_{\mathrm{c}}\right)^{3}$, and $h=$ $\left[10^{4} \mathrm{~W} / \mathrm{K} \mathrm{m}^{2}\right]\left(T / T_{\mathrm{c}}\right)^{3}$. We set $n_{1}=20$ and restrict $n(T)$ to $n \leqslant 100$. The sample dimensions are $a=1.3 \mathrm{~mm}$ and $d=100 \mathrm{~nm}$, and the applied magnetic field is ramped at the constant rate of $\dot{H}_{\mathrm{a}}=2.4 \times 10^{-6} j_{\mathrm{c} 0} \rho_{0} / a \mu_{0}$, from initially $H_{\mathrm{a}}=0$ and $T=T_{0} \equiv 0.2 T_{\mathrm{c}}$.

As the applied field gradually increases, shielding currents are induced in the sample, giving it a magnetic moment, $m$. Figure 3 displays the moment as a function of field, and shows that at low fields the magnitude of $m$ increases monotonously, as expected from the growing induced currents. Soon, the sheet current near the edge reaches the critical value $J_{\mathrm{c}}$, and magnetic

a

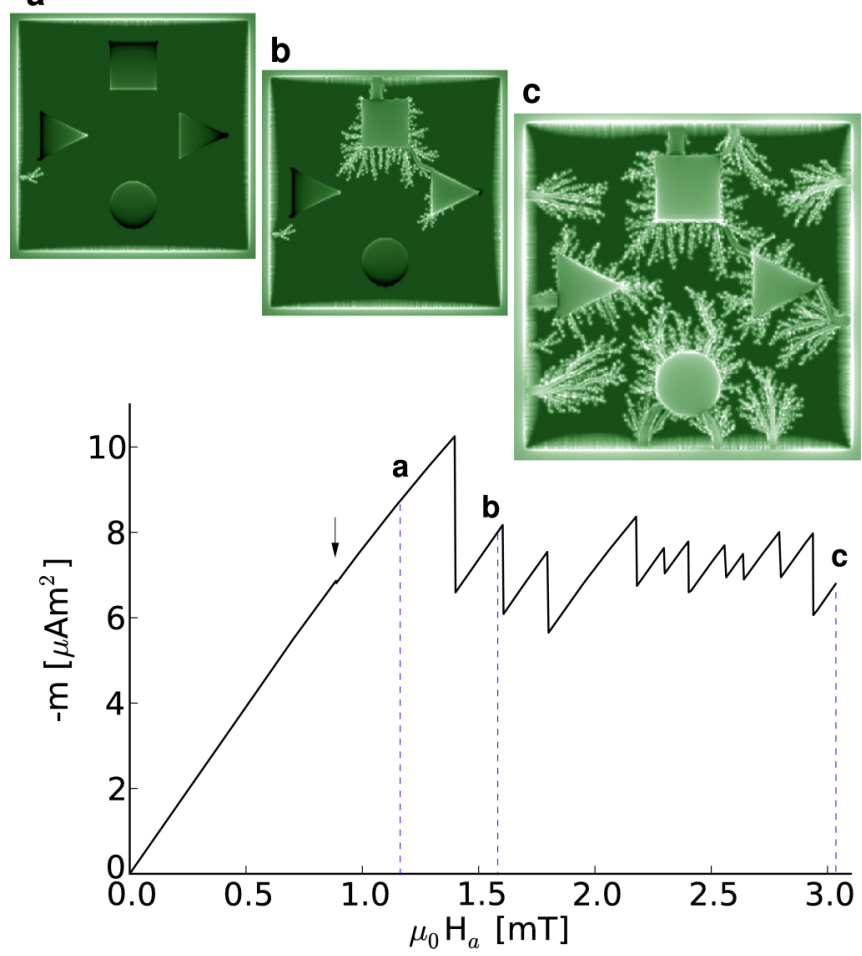

FIG. 3. (Color online) Magnetic moment as a function of applied field obtained by numerical simulation. Each drop in the curve corresponds to an avalanche event. The arrow marks the first avalanche. The images (a)-(c) show the $B_{\mathrm{z}}$ distribution at applied fields of $1.2,1.55$, and $3.05 \mathrm{mT}$, respectively. 


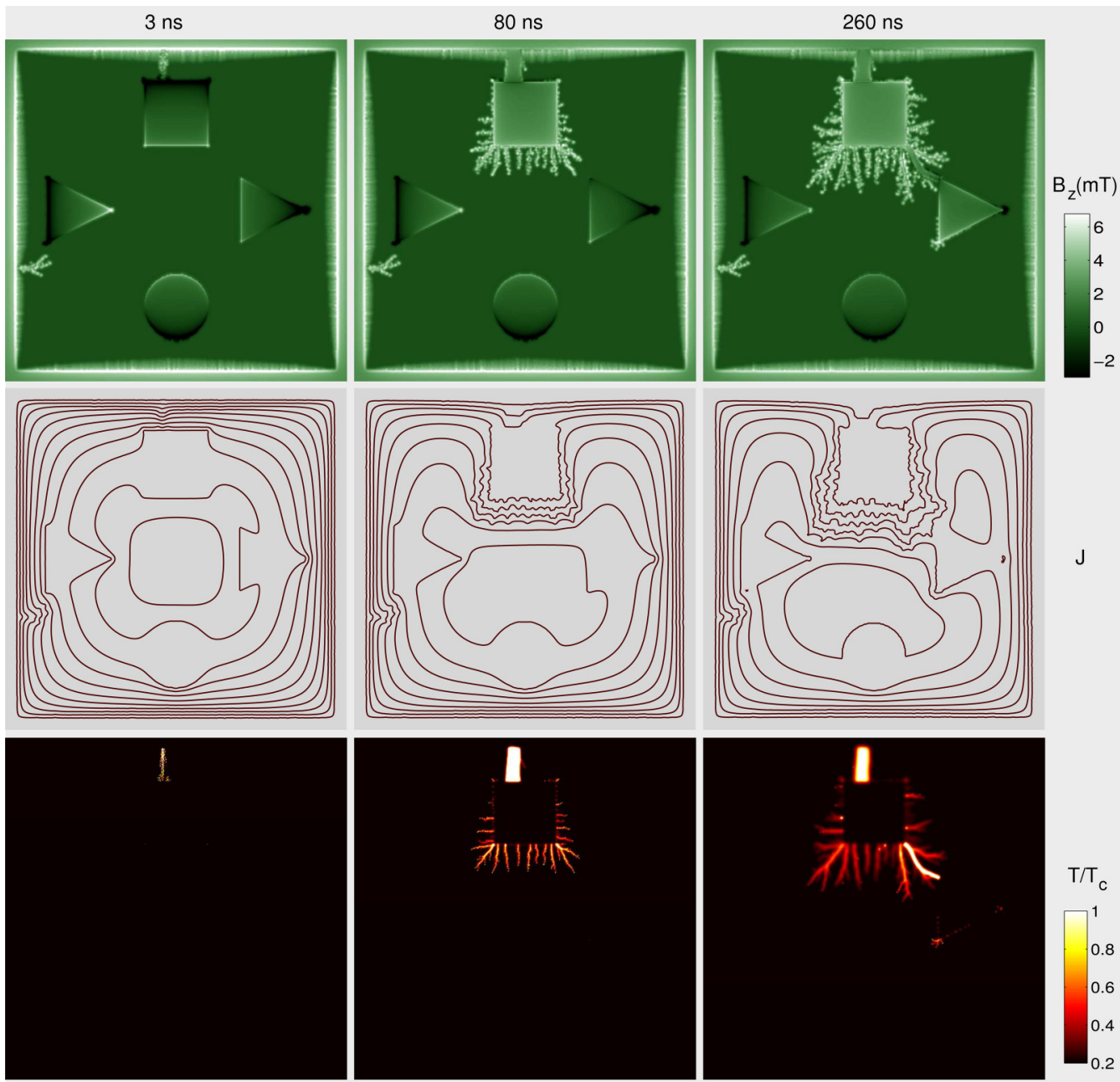

FIG. 4. (Color online) Numerical simulations of the avalanche occurring at $\mu_{0} H_{\mathrm{a}}=1.4 \mathrm{mT}$. Horizontal rows show the flux distribution, $B_{\mathrm{z}}$ (upper), current stream lines (middle), and temperature (lower) at times 3, 80, and $260 \mathrm{~ns}$ after nucleation of the event.

flux starts to enter the sample along the rim. At the field of $0.8 \mathrm{mT}$ a minor avalanche strikes near one of the triangular holes. The event is clearly visible in the flux distribution of image (a), but is hardly noticeable in the magnetic moment curve.

The next event occurs at $\mu_{0} H_{\mathrm{a}}=1.4 \mathrm{mT}$, and is a much bigger avalanche, as $m$ here makes a large drop. Image (b) shows that this avalanche alters the flux distribution significantly, and has filled both the square and triangular holes on the right side with sizable amounts of flux. Thereafter, the magnetization curve shows that avalanche events continue to appear with irregular intervals and magnitudes. The simulation was ended at the applied field of $3.05 \mathrm{mT}$, with the flux distributed as seen in image (c). During this field ramp a total of 11 avalanches took place, and the sequence of images of $B_{z}$ reproduces most features of the shown experimental images.

To illustrate the time evolution of an avalanche, Fig. 4 shows snapshots from the simulations, recorded at three moments in time after nucleation of the event at $\mu_{0} H_{\mathrm{a}}=1.4 \mathrm{mT}$. The figure presents maps of the flux density $B_{\mathrm{z}}$, the stream lines of the sheet current $J$, and the local temperature $T$. From the left column, one sees that already after 3 ns, a narrow channel of moving flux, accompanied by elevated temperature, is connecting the square hole to the flux reservoir outside the edge.

After 80 ns (middle column) this channel has grown in width, and the flux traffic has increased significantly. The $T$ map shows that in the entire channel the temperature now rises well above $T_{\mathrm{c}}$. Via this opening the square hole becomes filled with flux, which in turn nucleates secondary avalanches from the edge of the hole. The corresponding $J$ map confirms that a massive rearrangement of currents is taking place. Actually, at this stage the hole is more part of the outside than the inside of the superconductor, as far as shielding is concerned.

After $260 \mathrm{~ns}$ (right column) the secondary avalanches have penetrated deeper. In fact, one branch has reached another hole, namely, the triangular one on the right side. That hole is now being filled with flux via the narrow channel appearing as very bright in the $T$ map.

A more quantitative illustration of the interaction between avalanches and holes is presented in Fig. 5. The two graphs display the total flux $\Phi_{h}$ entering the square and triangular holes as functions of time during the avalanche occurring at $\mu_{0} H_{\mathrm{a}}=1.4 \mathrm{mT}$. First, flux is rapidly injected into the square hole with an almost constant rate, while the flux inside the triangular hole stays constant. At around $t=200 \mathrm{~ns}$ the flux 


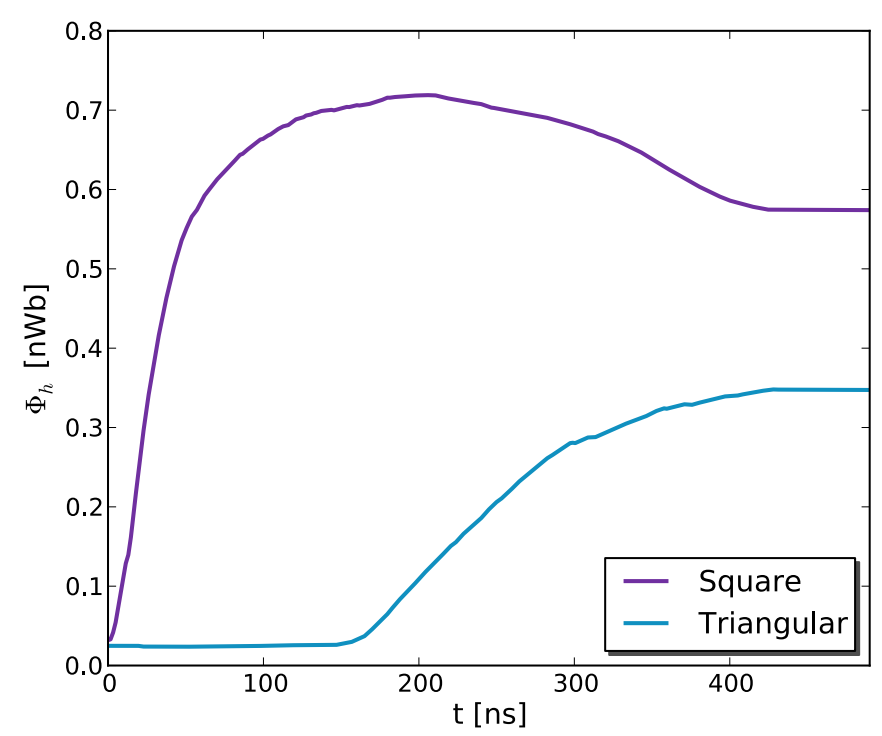

FIG. 5. (Color online) Total flux in the square and the triangular hole as functions of time during the avalanche at $\mu_{0} H_{\mathrm{a}}=1.4 \mathrm{mT}$.

in the square hole reaches a maximum. This occurs nearly at the same time as the flux in the triangular hole begins to increase. After $400 \mathrm{~ns}$ both curves reach a plateau as the avalanche comes to rest.

In the initial stage of the avalanche, the rate of change of the flux in the square hole is $\dot{\Phi}_{\mathrm{h}}=0.012 \mathrm{~Wb} / \mathrm{s}$. This represents the flux entering the hole while the channel to the external rim is heated above $T_{c}$. From Fig. 4 one can estimate that the channel width is $l=25 \mu \mathrm{m}$, which means that in the channel the electric field amounts to $E=\dot{\Phi}_{\mathrm{h}} / l=500 \mathrm{~V} / \mathrm{m}$. For comparison, an estimate of the electric field at the sample's outer edge created by the regular flux penetration gives $E=\mu_{0} \dot{H}_{\mathrm{a}} a=1.4 \mathrm{mV} / \mathrm{m}$. Note that in the present model no heat flows in the substrate below the holes. This suggests that the electrodynamics alone can lead to avalanche cascades between holes in a superconducting film.

In summary, the present work has shown that thermomagnetic avalanches in superconducting films tend to be attracted by holes in the film. The numerical simulations reveal the detailed dynamics at the various stages of an avalanche as it propagates from one hole to another. Typically, a hole becomes filled with flux via a narrow normal-state channel created between the film's external edge and the hole. It is found that this often leads to secondary avalanches, which spread to neighboring holes or parts of the film not yet penetrated by flux. Holes with sharp corners, such as square and triangular holes, are those that most frequently create avalanche cascades.

The samples were grown in Laboratório de Conformação Nanométrica (LCN-IF-UFRGS), and the lithography was made in Laboratório de Microfabricação (LMF/LNNano/CNPEM). The work was partially supported by the Sao Paulo Research Foundation (FAPESP) Grant No. 2013/16.097-3, the Brazilian National Council for Scientific and Technological Development (CNPq), the Brazilian program Science without Borders, as well as the CAPES-SIU-2013/10046 project "Complex fluids in confined environments."
[1] C. P. Bean, Rev. Mod. Phys. 36, 31 (1964).

[2] M. R. Wertheimer and J. le G. Gilchrist, J. Phys. Chem. Solids 28, 2509 (1967).

[3] P. Leiderer, J. Boneberg, P. Brüll, V. Bujok, and S. Herminghaus, Phys. Rev. Lett. 71, 2646 (1993).

[4] W. DeSorboand V. L. Newhouse, J. Appl. Phys. 33, 1004 (1962).

[5] G. J. Dolan, J. Low Temp. Phys. 15, 111 (1973).

[6] C. A. Durán, P. L. Gammel, R. E. Miller, and D. J. Bishop, Phys. Rev. B 52, 75 (1995).

[7] T. H. Johansen, M. Baziljevich, D. V. Shantsev, P. E. Goa, Y. M. Galperin, W. N. Kang, H. J. Kim, E. M. Choi, M.-S. Kim, and I. Lee, Europhys. Lett. 59, 599 (2002).

[8] I. A. Rudnev, S. V. Antonenko, D. V. Shantsev, T. H. Johansen, and A. E. Primenko, Cryogenics 43, 663 (2003).

[9] E. Altshuler, T. H. Johansen, Y. Paltiel, P. Jin, K. E. Bassler, O. Ramos, Q. Y. Chen, G. F. Reiter, E. Zeldov, and C. W. Chu, Phys. Rev. B 70, 140505 (2004).

[10] S. C. Wimbush, B. Holzapfel, and Ch. Jooss, J. Appl. Phys. 96, 3589 (2004).

[11] I. A. Rudnev, D. V. Shantsev, T. H. Johansen, and A. E. Primenko, Appl. Phys. Lett. 87, 042502 (2005).

[12] M. Motta, F. Colauto, W. A. Ortiz, J. Fritzsche, J. Cuppens, W. Gillijns, V. Moshchalkov, T. H. Johansen, A. Sanchez, and A. V. Silhanek, Appl. Phys. Lett. 102, 212601 (2013).

[13] M. Baziljevich, E. Baruch-El, T. H. Johansen, and Y. Yeshurun, Appl. Phys. Lett. 105, 012602 (2014).
[14] R. G. Mintsand A. L. Rakhmanov, Rev. Mod. Phys. 53, 551 (1981).

[15] R. G. Mintsand E. H. Brandt, Phys. Rev. B 54, 12421 (1996).

[16] D. V. Denisov, D. V. Shantsev, Y. M. Galperin, E.-M. Choi, H.-S. Lee, S.-I. Lee, A. V. Bobyl, P. E. Goa, A. A. F. Olsen, and T. H. Johansen, Phys. Rev. Lett. 97, 077002 (2006).

[17] J. Albrecht, A. T. Matveev, J. Strempfer, H.-U. Habermeier, D. V. Shantsev, Y. M. Galperin, and T. H. Johansen, Phys. Rev. Lett. 98, 117001 (2007).

[18] I. Aranson, A. Gurevich, and V. Vinokur, Phys. Rev. Lett. 87, 067003 (2001).

[19] I. S. Aranson, A. Gurevich, M. S. Welling, R. J. Wijngaarden, V. K. Vlasko-Vlasov, V. M. Vinokur, and U. Welp, Phys. Rev. Lett. 94, 037002 (2005).

[20] J. I. Vestgården, D. V. Shantsev, Y. M. Galperin, and T. H. Johansen, Phys. Rev. B 84, 054537 (2011).

[21] J. I. Vestgården, D. V. Shantsev, Y. M. Galperin, and T. H. Johansen, Sci. Rep. 2, 886 (2012).

[22] J. Albrecht, A. T. Matveev, M. Djupmyr, G. Schütz, B. Stuhlhofer, and H. Habermeier, Appl. Phys. Lett. 87, 182501 (2005).

[23] P. Mikheenko, A. J. Qviller, J. I. Vestgården, S. Chaudhuri, I. J. Maasilta, Y. M. Galperin, and T. H. Johansen, Appl. Phys. Lett. 102, 022601 (2013).

[24] P. Mikheenko, T. H. Johansen, S. Chaudhuri, I. J. Maasilta, and Y. M. Galperin, Phys. Rev. B 91, 060507(R) (2015). 
[25] V. Vlasko-Vlasov, U. Welp, V. Metlushko, and G. W. Crabtree, Physica C 341-348, 1281 (2000).

[26] M. Menghini, R. J. Wijngaarden, A. V. Silhanek, S. Raedts, and V. V. Moshchalkov, Phys. Rev. B 71, 104506 (2005).

[27] M. Motta, F. Colauto, R. Zadorosny, T. H. Johansen, R. B. Dinner, M. G. Blamire, G. W. Ataklti, V. V. Moshchalkov, A. V. Silhanek, and W. A. Ortiz, Phys. Rev. B 84, 214529 (2011).

[28] M. Motta, F. Colauto, J. I. Vestgården, J. Fritzsche, M. Timmermans, J. Cuppens, C. Attanasio, C. Cirillo, V. V. Moshchalkov, J. Van de Vondel, T. H. Johansen, W. A. Ortiz, and A. V. Silhanek, Phys. Rev. B 89, 134508 (2014).

[29] Åge Andreas Falnes Olsen, T. H. Johansen, D. Shantsev, E.-M. Choi, H.-S. Lee, H. J. Kim, and S.-I. Lee, Phys. Rev. B 76, 024510 (2007).
[30] F. Colauto, J. I. Vestgården, A. M. H. de Andrade, A. A. M. Oliveira, W. A. Ortiz, and T. H. Johansen, Appl. Phys. Lett. 103, 032604 (2013).

[31] L. E. Helseth, A. G. Solovyev, R. W. Hansen, E. I. Il'yashenko, M. Baziljevich, and T. H. Johansen, Phys. Rev. B 66, 064405 (2002).

[32] M. Baziljevich, T. H. Johansen, H. Bratsberg, Y. Shen, and P. Vase, Appl. Phys. Lett 69, 3590 (1996).

[33] See Supplemental Material at http://link.aps.org/supplemental/ 10.1103/PhysRevB.92.144510 for MOI and simulated videos showing the dynamics.

[34] J. I. Vestgården, P. Mikheenko, Y. M. Galperin, and T. H. Johansen, New J. Phys. 15, 093001 (2013). 